\title{
Evaluación sobre los procesos formativos de los docentes de la región de Magallanes (Chile)
}

The evaluation of the formative processes in teachers from the Magallanes region (Chile)

\author{
Mario Garay Aguilar ${ }^{a}$ \& Mario Sillard Velásouez ${ }^{b}$ \\ ${ }^{a}$ Doctor en Educación. Coordinador del Observatorio de Políticas Educativas y \\ Prácticas Pedagógicas, Universidad de Magallanes \\ b Sociólogo, Investigador en Observatorio de Políticas Educativas y \\ Prácticas Pedagógicas, Universidad de Magallanes. \\ $\measuredangle$ mario.garay@umag.cl; mario.sillard@umag.cl
}

\begin{abstract}
RESUMEN
En las últimas décadas se han diseñado e implementado en nuestro país diversas políticas orientadas a mejorar la Formación Inicial Docente (FID). Para conocer en qué medida estas acciones han tenido efectos, en los docentes en ejercicio de la Región de Magallanes, en el presente artículo se investigó la percepción de los profesores sobre cómo fue su formación inicial, qué de ella les ha sido útil en su desempeño profesional y qué carencias identifican al ejercer su profesión en las escuelas. Los hallazgos muestran en general un alto nivel de satisfacción con la FID y que los docentes más jóvenes evalúan de forma más crítica la casa de estudios de la que egresaron y los profesores que egresaron de instituciones selectivas reportan una positiva evaluación sobre sus procesos de formación.
\end{abstract}

PALABRAS CLAVE: Carrera Docente, Formación Pedagógica, Formación Inicial Docente, Procesos Formativos, Trayectorias Académicas.

\begin{abstract}
In the last decades, diverse policies aimed at improving initial teacher training (FID) have been designed and implemented in our country. In order to know to what extent these actions have had effect in the teachers currently in service in the Region of Magallanes, this investigation was based on the teachers' perception on their own initial teaching training,
\end{abstract}


what it has been useful in their professional performance and what deficiencies they identify while they are in-service teachers at schools. The findings show, in general, a high level of satisfaction with the teaching training formation (FID) and younger teachers give a more critical evaluation to the house of studies where they graduated from than those who were graduated from selective institutions who report a positive evaluation on their training processes.

KEY WORDS: Teaching Career, Pedagogical Training, Initial Teacher Training, Formative Processes, Academic Trajectories.

\section{INTRODUCCIÓN}

La Formación Inicial Docente (FID) es uno de los factores más preponderantes a la hora del desempeño del docente (Conway, 2013) y que requiere, por tanto, de una seria revisión, sobre todo si se desarrolla en un sistema educacional marcado por una profunda desigualdad y segmentación socioeconómica como es el caso chileno. Dentro de ese marco, resulta de interés abordar la percepción de la calidad respecto a la formación que tienen los docentes que hoy están en ejercicio laboral en establecimientos educativos. En especial, cuando se mantienen los bajos resultados en la Evaluación Docente a profesores que ejercen en el sector municipal y los discretos resultados de la prueba INICIA aplicada a docentes recién egresados de pedagogía (Ruffinelli, 2013).

Lo anterior ha generado la discusión de cómo garantizar la calidad de las carreras de pedagogías (Manzi et al. 2011). Además, la evidencia nos indica que los docentes recién egresados son actores que han recibido una escasa atención en el ámbito de la investigación educacional (Cisternas, 2011), pero que progresivamente se han ido consolidando como línea de investigación (Gorichon et al. 2015).

Por otra parte, la preocupación por la calidad de la FID, explica el desarrollo de una serie de políticas y acciones destinadas a corregir los problemas observados desde mediados de los años noventa hasta hoy (Avalos, 2014). De ahí que surgen iniciativas tales como el Programa de Fortalecimiento de la Formación Inicial Docente (FFID), -implementado a fines de los años noventa y que posibilitó, entre otras cosas, la instauración practicas pedagógicas progresivas-, los proyectos MECESUP, el Sistema de Acreditación de Carreras, la Prueba Inicia, la elaboración de estándares orientadores y la implementación de convenios de desempeño para la FID. Si bien estas iniciativas han aportado a la formación de profesores, aún queda mucho por avanzar entre las instituciones formadoras y el sistema escolar (Ruffinelli, 2013). Por tal motivo, es necesario integrar los resultados de las investigaciones e innovaciones educativas que se realizan en los contextos socioeducativos, para ir eliminando la disociación que tradicionalmente se ha planteado entre teoría y práctica (Díaz et al. 2015). Esto aportaría al proceso de renovación curricular que se inició en distintas universidades a través de la 
adjudicación de Convenios de Desempeño, los cuales buscan que las instituciones formen a "los profesores del siglo XXI que Chile necesita, con competencias profesionales de alto nivel que generen cambios notables en la calidad del aprendizaje en las aulas escolares" (MINEDUC, n.d).

La clave de la FID es que la existencia de profesores bien preparados puede ayudar a cerrar la brecha de desempeño entre los estudiantes de estratos socioeconómicos altos y bajos, ayudando así a los estudiantes de bajo desempeño a nivelarse (OCDE, 2012). Esto aportaría a romper el "círculo vicioso" generado por la estructura segregadora del sistema educativo chileno, el cual tiene un doble proceso de selección; no sólo bajo el modelo de selección de establecimiento educativo a través de las condiciones socioeconómicas en base al pago de arancel, sino que además induce a que docentes recién egresados retornen a trabajar a establecimientos similares a aquellos de los cuales egresaron (Ruffinelli, 2013; Puga et al. 2015). Lo anterior, provoca que el contexto de origen socioeconómico del docente sea predictor del tipo de establecimiento donde se desempeñará después laboralmente. Esto es parte de un círculo virtuoso-vicioso, donde la atracción del talento está directamente relacionada con los mecanismos de selección y la proyección social de la profesión (Park \& Byun, 2015).

Sin embargo, más allá del nivel socioeconómico de proveniencia o de ejercicio profesional de los docentes, es importante hacer foco en la relación de los estudiantes de pedagogía con sus procesos de formación y, en especial, con las herramientas con las que contaría este futuro docente para desenvolverse en el contexto laboral. En este sentido, se asume que “aprender a enseñar es un proceso activo y constructivo, en que el aprendizaje está situado en contextos y culturas, donde el futuro docente aprende y juega un papel importante en el proceso de aprendizaje de tal forma que es un constructor activo de conocimiento que da sentido al mundo interpretando las experiencias a través de sus conocimientos previos" (Marcelo, 2009, p. 40). Esto implica que los estudiantes de pedagogía no sólo se expongan al aprendizaje de contenidos teóricos o disciplinares sino que también puedan acceder a culturas organizacionales escolares que les permitan como futuros docentes, dimensionar posibilidades de enfrentar sus miedos, vivenciar el trabajo colectivo, la autonomía profesional y la autogestión, entre otras acciones. Lo anterior debe realizarse a través de un trabajo conjunto y responsabilidad compartida entre las universidades formadoras de la FID y los docentes que trabajan en las escuelas. Esta modalidad de trabajo hará posible definir una agenda de transformación mutua al servicio de una educación con equidad y calidad para todos (Darling-Hammond, 2006; Musset, 2010).

En este sentido, investigaciones recientes, rescatan varios puntos referenciados por docentes en ejercicio en torno a los insuficiencias que vivieron en sus puestos de trabajo (Gaete et al. 2016). Destacan, entre los principales problemas: las áreas del trabajo administrativo, metodología de la enseñanza, dominio de grupo, atención a la diversidad, evaluación, relación con los apoderados y problemáticas a nivel de relación escuela-familia. 
Esto plantea los desafíos y debates para la construcción de una base de investigación que informe, oriente y/o justifque determinadas políticas y prácticas de formación, aportando con respuestas contextualizadas y pertinentes a las particulares realidades de los sistemas educativos (Borko et al. 2008). En este escenario la investigación y la vinculación de las carreras de pedagogía con los establecimientos educacionales son aspectos claves para mejorar la FID (Díaz et al. 2015).

Para que esas modificaciones a la formación docente tenga efecto sustantivo, es preciso además, que éstas vengan desde la opinión y el diagnóstico interactivo entre docentes en ejercicio y universidades formadoras de profesores y profesoras. Aunque la evidencia tanto nacional como internacional señale que esta es una alianza compleja que sobrepasa voluntades institucionales (NCATE, 2010).

En ese sentido, la presente investigación busca aportar en la discusión sobre la FID rescatando la opinión y evaluación de los docentes respecto a sus ciclos formativos, lo que sirve de base para la formación de profesores y como insumo para cursos de acción en educación continua, siendo uno de los criterios que definen la calidad de la educación (García \& Castro, 2012). En este escenario se "justica la necesidad de escuchar, recoger y valorar la voz del profesorado para conocer las fortalezas y limitaciones de los planes de formación y poder mejorar su competencia profesional, que sin duda, continúa siendo un desafío para todos" (Díaz et al. 2015, p. 243).

\section{METODOLOGÍA}

Esta investigación aplicó un enfoque cuantitativo descriptivo, por ser un método utilizado en diferentes ámbitos, desde estudios de opinión hasta diagnósticos para establecer políticas educativas. El objetivo principal de este enfoque, es conocer la distribución de ciertas variables de interés en una población determinada (Hueso \& Cascanti, 2012), a través de una medición estandarizada y numérica, características propias de las ciencias exactas (Hernández, 2003). Para el análisis, en tanto, se adoptó un diseño correlacional y transversal.

Se aplicó un criterio de encuestar a todos los/as profesionales que ejercieran docencia en el aula y/o tuvieran título de pedagogía, contratados por algún establecimiento educacional, incluyendo aquellos que ocupen cargos directivos. Según datos del MINEDUC, en el año 2016 la planta docente de la región de Magallanes alcanzaba las 2.680 personas. El muestreo se intencionó en las comunas de Punta Arenas y Puerto Natales (en donde trabaja el $98 \%$ del total de los docentes). Se aplicó el trabajo de campo durante los meses agosto y septiembre 2016, recorriendo todos los establecimientos de ambas comunas instando a que voluntariamente, los docentes contestaran el instrumento. Finalmente, 75 establecimientos accedieron a participar del estudio, lo que se tradujo en 1.066 docentes dispuestos a contestar el instrumento, los que se segmentan de la siguiente manera: Educación Inicial: 
11,4\%, Educación Municipal: 43,9\%, Establecimientos Particulares Subvencionados: 32,2\% y establecimientos Particulares Pagados: 12,5\%.

El instrumento, en formato de encuesta auto-aplicada, contiene tres partes. La primera, sobre antecedentes personales y respecto a sus condiciones laborales; la segunda, sobre trayectoria y expectativa laboral, y la última, relacionada con la evaluación sobre sus procesos de formación. Esta tercera parte del instrumento es la que otorga la mayor información para este estudio y específicamente consistió en consultar a los docentes mediante escala Likert, cómo evaluaban distintas áreas de su proceso formativo considerando la Universidad en donde habían estudiado. El instrumento fue validado a través de juicio experto por docentes tanto del medio escolar como del ámbito académico.

\section{RESULTADOS}

Considerando la gran cantidad de instituciones de educación superior que surgieron de los encuestados (más de 80, muchas de ellas, ya no existentes), se optó por ordenarlas en base a su prestigio institucional, pues se considera un indicador estable que pueda diferenciarlas y ordenarlas en base a su nivel de selectividad.

De esta manera, y con el fin de favorecer los análisis, se segmentaron las instituciones de educación superior con más de 5 años de acreditación institucional de las demás, las primeras serán consideradas como "Selectivas" y las universidades con 5 años o menos de acreditación serán consideradas como "No-Selectivas".

A continuación, se presentan, los resultados sobre la caracterización de los docentes en relación a los elementos facilitadores u obstaculizadores que vivenciaron en su formación inicial. Luego, se describen los resultados de cada uno de los ítems en la totalidad de los docentes encuestados, mostrando luego la valoración de los actores con respecto a la FID y finalmente, se segmentan estos resultados por categorías de selectividad y años de los docentes.

La Fig. 1, muestra la distribución de las respuestas de todos los/as docentes en las dimensiones relativas a su formación inicial.

Como se muestra en todos los ítems consultados, la gran mayoría de los encuestados manifiesta como muy bueno-bueno el proceso de su formación como docente en las distintas dimensiones. La evaluación de la preparación en TIC y en herramientas didácticas aparece como el área más crítica, donde más de un $40 \%$ de los encuestados indica que su formación ha sido regular o mala.

A continuación se puede apreciar (Fig. 2) la distribución de la segmentación de las respuestas de los/as docentes por universidades Selectivas y No-Selectivas, en relación a las condiciones de infraestructura en las que se desarrolló su formación inicial y al mismo tiempo comprender mejor las valoraciones respecto a sus procesos formativos. 


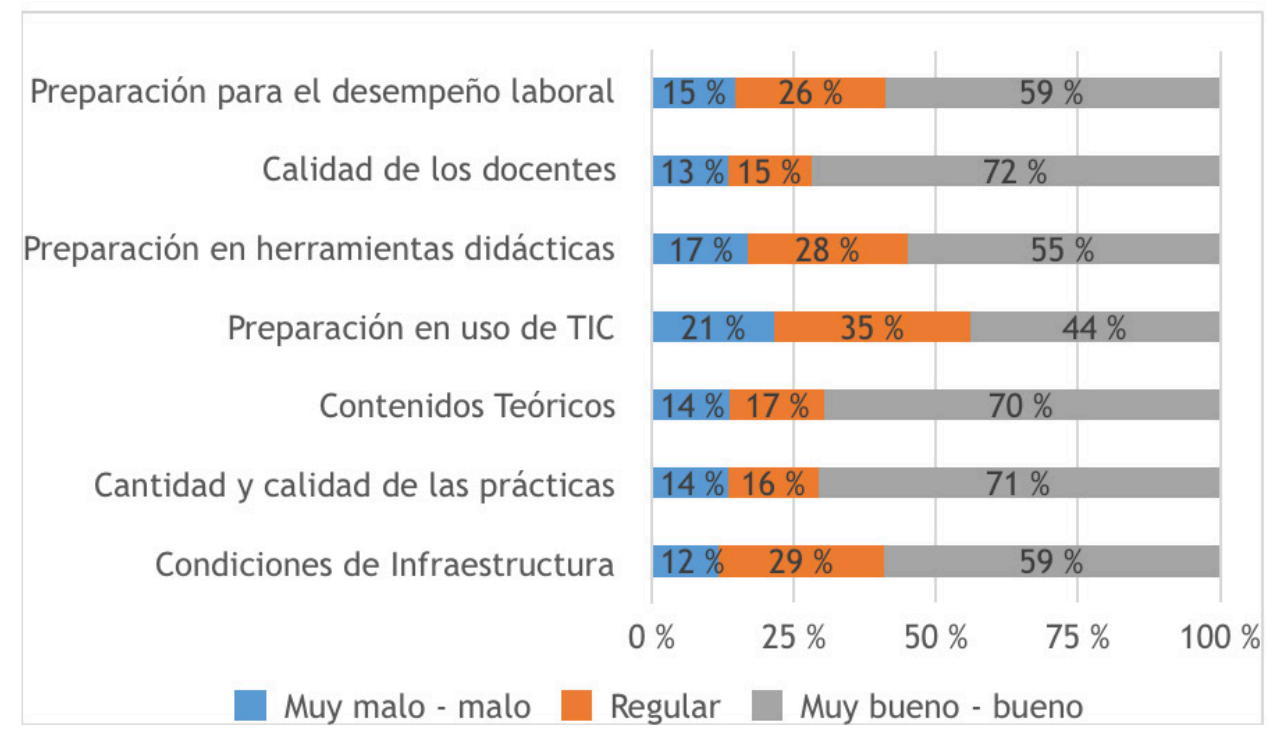

Fig. 1. Evaluación por ítem de procesos formativos por parte de la totalidad de los docentes encuestados.

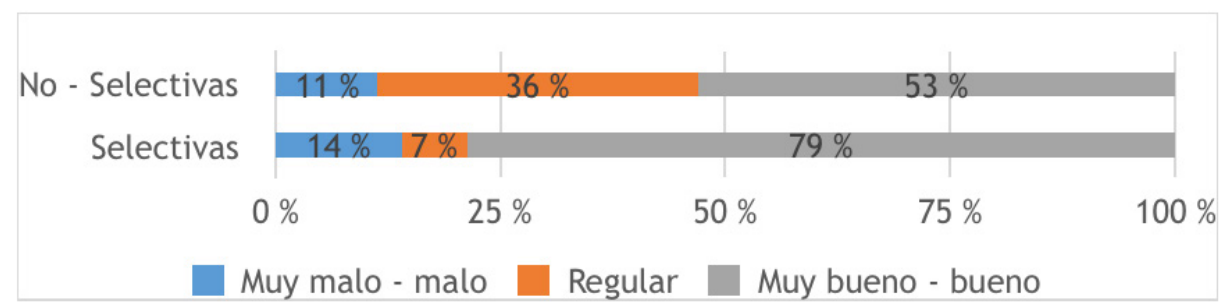

Fig 2. Evaluación de procesos formativos según Universidad de egreso. Ítem: condiciones de infraestructura.

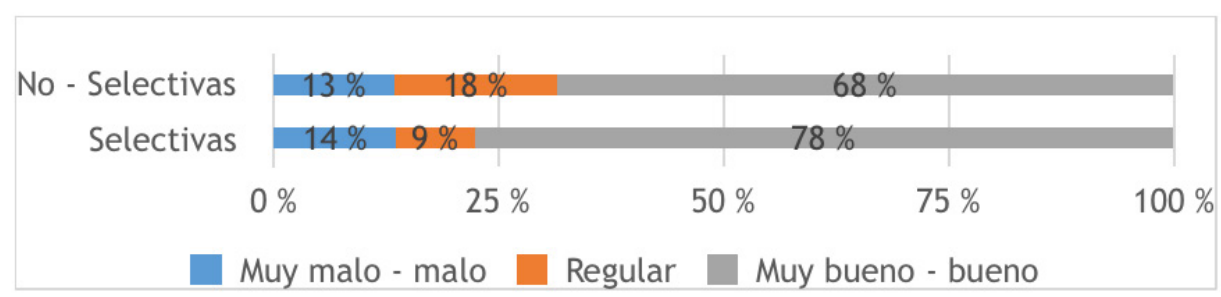

Fig. 3. Evaluación de procesos formativos según Universidad de egreso. Ítem: cantidad y calidad de las prácticas.

Se logra apreciar, en algunos de los ítems hay bastante variación entre los docentes según su universidad de egreso. En todos los casos, los egresados de las universidades No-Selectivas tienden a tener una evaluación menos positiva que sus colegas egresados de universidades Selectivas. Al evaluar infraestructura por ejemplo, cerca de la mitad de los docentes encuestados cree que las condiciones fueron regulares o malas, mientras que el 79\% los egresados de universidades Selectivas cree que la infraestructura era Buena o Muy Buena. 


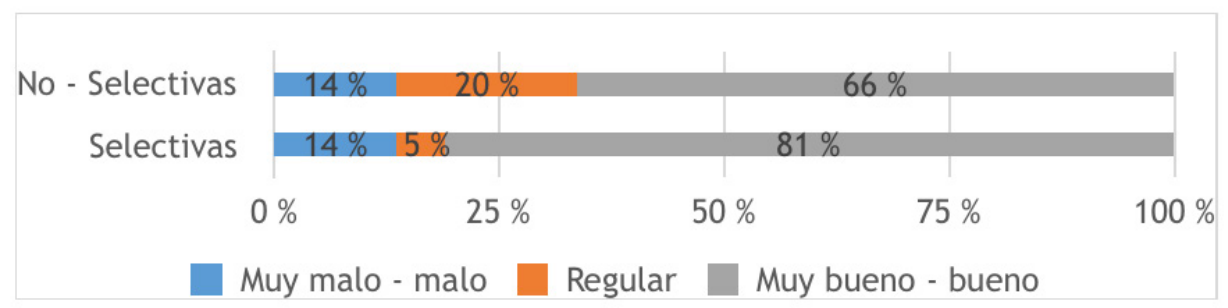

Fig. 4. Evaluación de procesos formativos según Universidad de egreso. Ítem: contenidos teóricos.

Cabe mencionar que al segmentar los resultados para "cantidad y calidad de las prácticas", "contenidos teóricos" (Fig. 4) o "la calidad de los docentes" (Fig. 7), las diferencias entre las universidades se mantienen, pero el nivel "Bueno - Muy Bueno" de ambas categorías es mayoritario. Es en las áreas que tienen que ver con el ejercicio práctico de la docencia más que en los insumos teóricos que los docentes contestan diferenciados en base al tipo de universidad al que asistieron

En el caso de la preparación en las TIC, los docentes de universidades No-Selectivas vuelven a ser más críticos, el 56\%, indica que su formación muy mala y/ regular. Aunque este ítem también está muy influenciado por el año de egreso de los docentes.

Ruffinelli (2013) publica un estudio similar (evaluación de procesos de formación de docentes) pero que sólo pregunta a docentes jóvenes (con 5 años o menos de egreso), donde también segmenta las universidades por nivel de selectividad aunque utilizando los puntajes PSU de ingreso a las carreras de pedagogía. En aquella investigación, a diferencia de la nuestra, los egresados de universidades Selectivas presentan pocas diferencias con los egresados de otras universidades e incluso cuando hay variación, los docentes de universidades Selectivas evalúan de forma más crítica su proceso formativo.

Para nuestro caso, en el ítem de "Preparación de las herramientas didácticas", vuelve a haber diferencias entre el tipo de universidad, en donde hay más proporción de docentes provenientes de universidades No-Selectivas que indican que la preparación fue regular o mala.

La preparación para el mundo laboral, es algo que también resienten los egresados de universidades No-Selectivas: un 47\% cree que fue regular o mala, mientras que 3 de cada 4 egresados de universidades Selectivas considera que su preparación en este ítem fue Buena o Muy Buena.

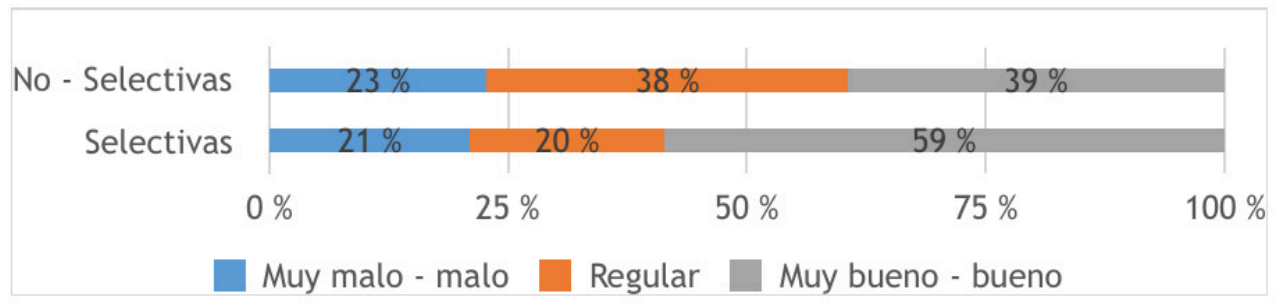

FIg. 5. Evaluación de procesos formativos según universidad de egreso. Ítem: preparación en las TIC 


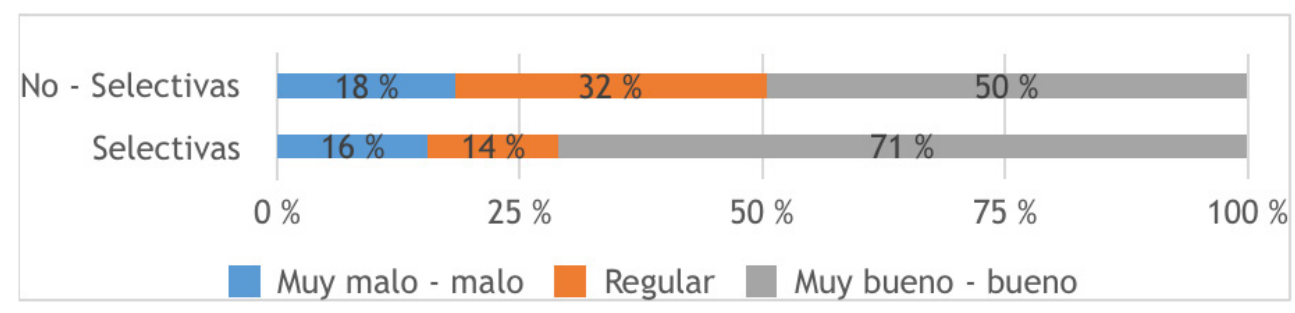

Fig. 6. Evaluación de procesos formativos según Universidad de egreso. Ítem: preparación en herramientas didácticas.

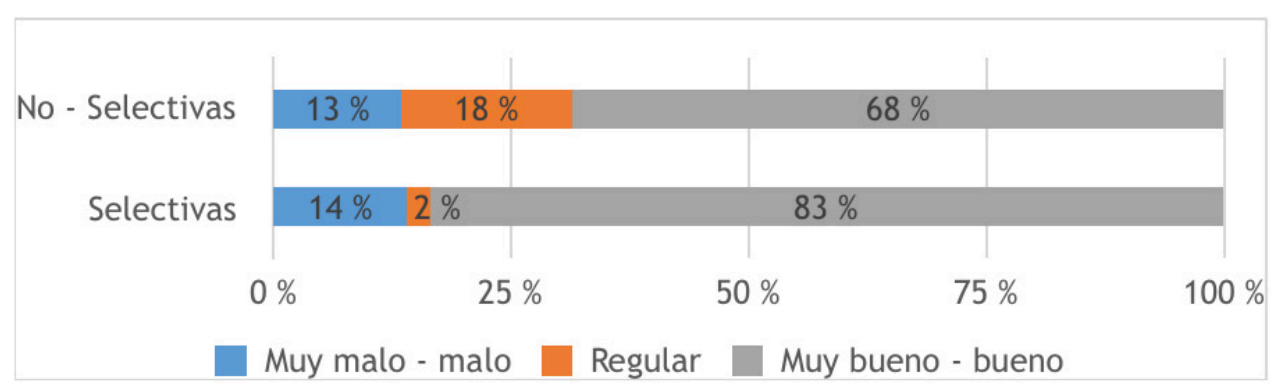

Fig. 7. Evaluación de procesos formativos según Universidad de egreso. Ítem: calidad de los docentes.

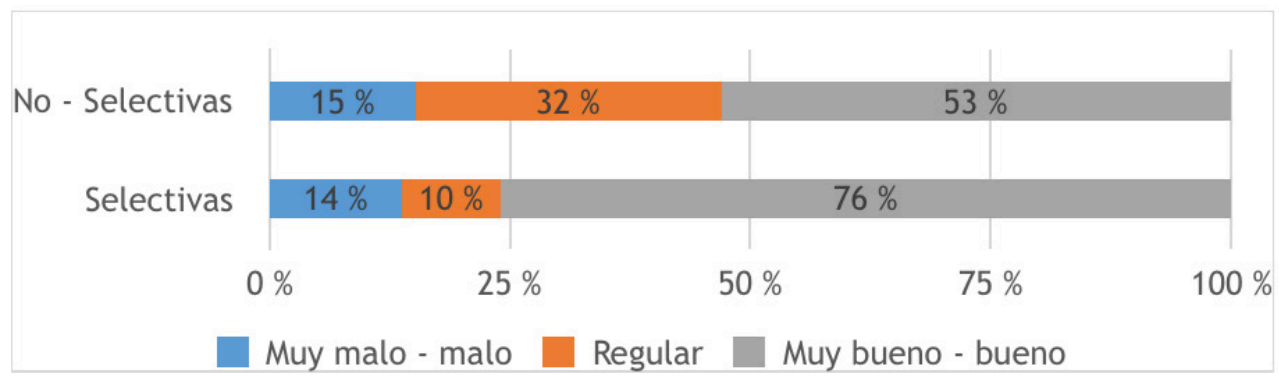

Fig. 8. Evaluación de procesos formativos según Universidad de egreso. Ítem: preparación para el mundo laboral.

A partir de la Fig. 9, las respuestas de los/as docentes encuestados se analizarán por edad. Como se puede apreciar, los docentes más jóvenes tienden a evaluar sus procesos de formación de manera menos positiva y más crítica que sus colegas que tienen más de 40 años de edad.

La dimensión infraestructura debiera ser de particular atención pues, intuitivamente, se podría predecir que los profesores que han egresado hace menos tiempo, debieron haber tenido acceso a universidades con más y mejor espacio físico para el desarrollo de procesos educativos que las generaciones más antiguas. Sin embargo, se detecta una clara tendencia más crítica de por parte de los docentes más jóvenes, con un $42 \%$ en la categorías muy malo-malo y regular. También se encuentran diferencias en la dimensión calidad y cantidad de contenidos teóricos. 


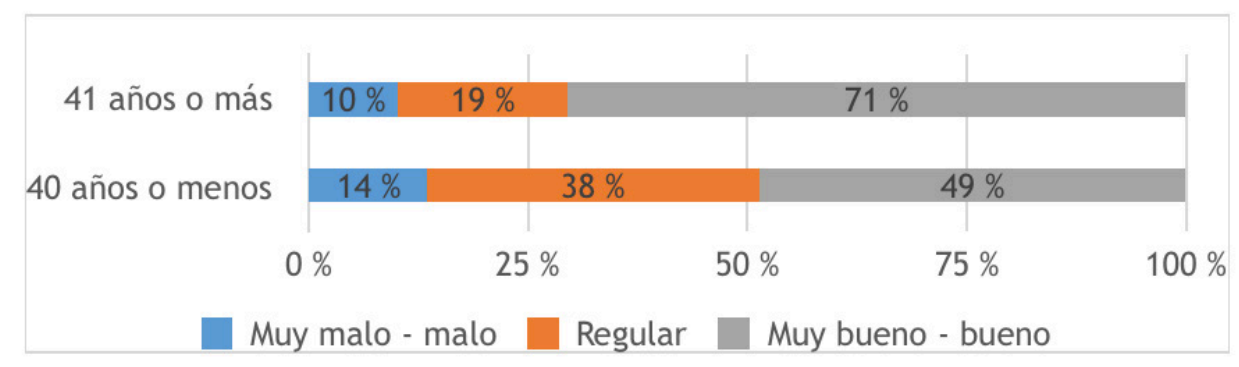

Fig. 9. Evaluación de procesos formativos segmentados por edad. Ítem: condiciones de infraestructura.

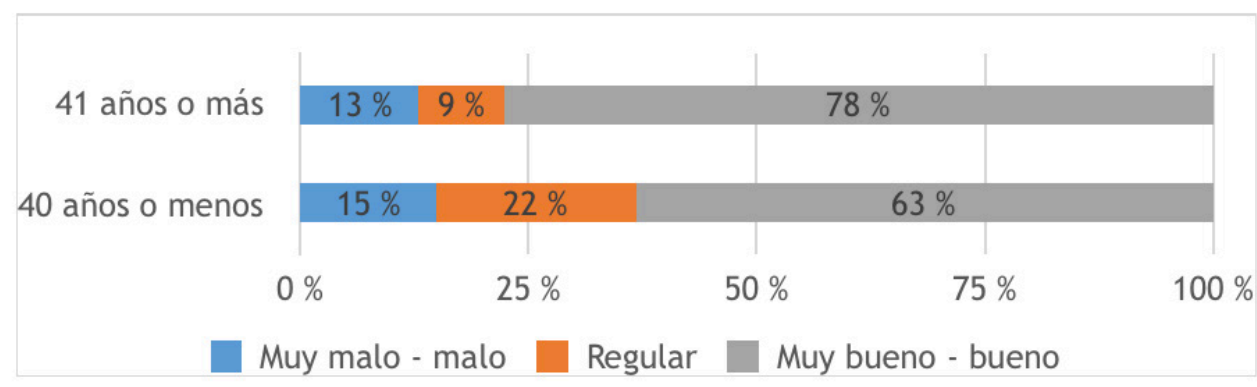

Fig. 10. Evaluación de procesos formativos segmentados por edad. Ítem: Calidad y cantidad de contenidos teóricos.

Se observa que los docentes con 41 años o más, tienen una visión más positiva en la calidad y cantidad de contenidos teóricos, con un 78\% entre muy bueno-bueno. En la dimensión relacionada con la formación práctica, se encuentran diferencias sustantivas por edad vinculadas con las herramientas pedagógicas didácticas o con la preparación para el mundo laboral.

Los docentes más experimentados evalúan mejor las herramientas didácticas, con un 63\% entre muy bueno-bueno.

En relación a la preparación para el mundo laboral que adquirieron en sus procesos de formación universitaria, ésta también es bien evaluada por los docentes de 41 años o más.

En la Fig. 12 los docentes de 40 años o menos, muestran un 47\% de valoración muy mala a regular.

Otra consulta realizada a los docentes fue cómo imaginaban su carrera profesional en 10 años más, habiendo cuatro alternativas posibles de contestar: "como docente en este u otro establecimiento", "como directivo de un establecimiento", "no ejerciendo como docente" y "jubilado". Excluyendo a los que en ese plazo piensan que van a jubilar, sus respuestas las segmentamos en función de la evaluación que hacen de sus procesos formativos.

Entonces, se podría predecir que quienes visualizan el futuro aún proyectados como docentes (de aula o como directivos), son aquellos que tienen una visión más favorable de 


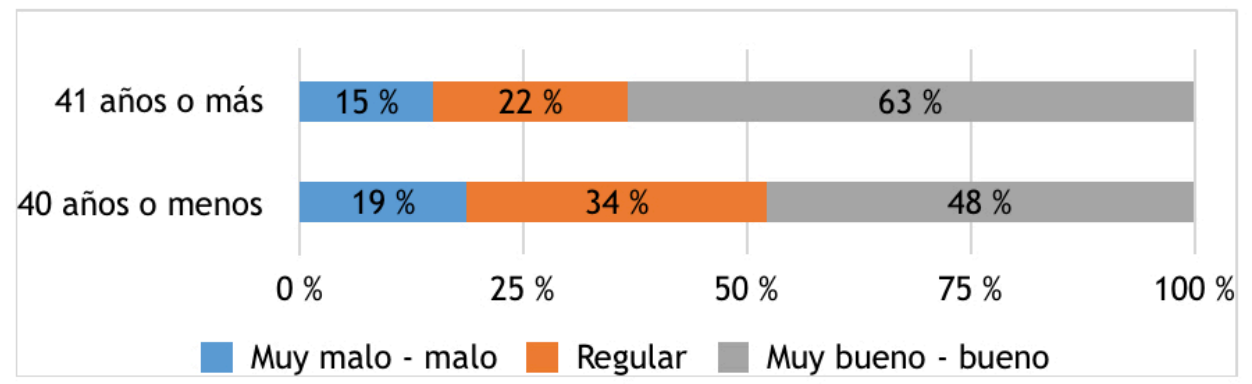

Fig. 11. Evaluación de procesos formativos segmentados por edad. Ítem: Preparación en herramientas didácticas.

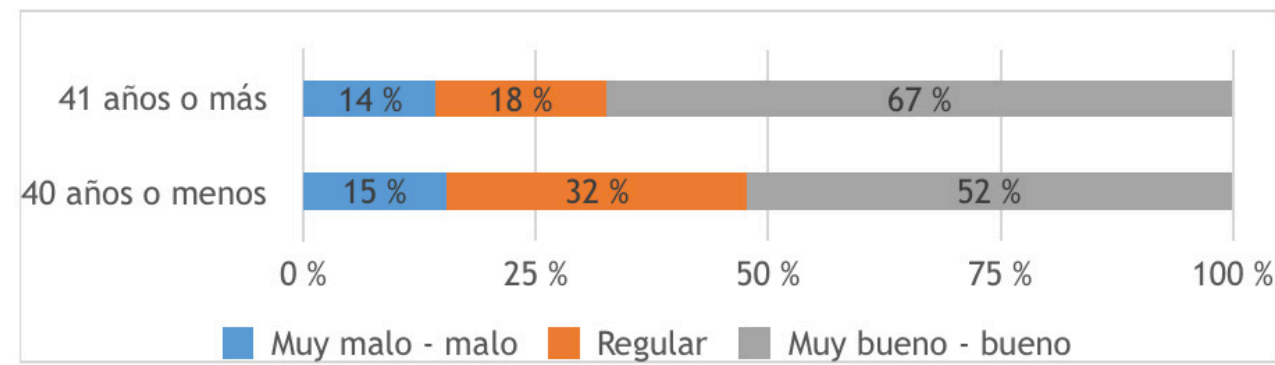

Fig. 12. Evaluación de procesos formativos segmentados por edad. Ítem: Preparación para el mundo laboral

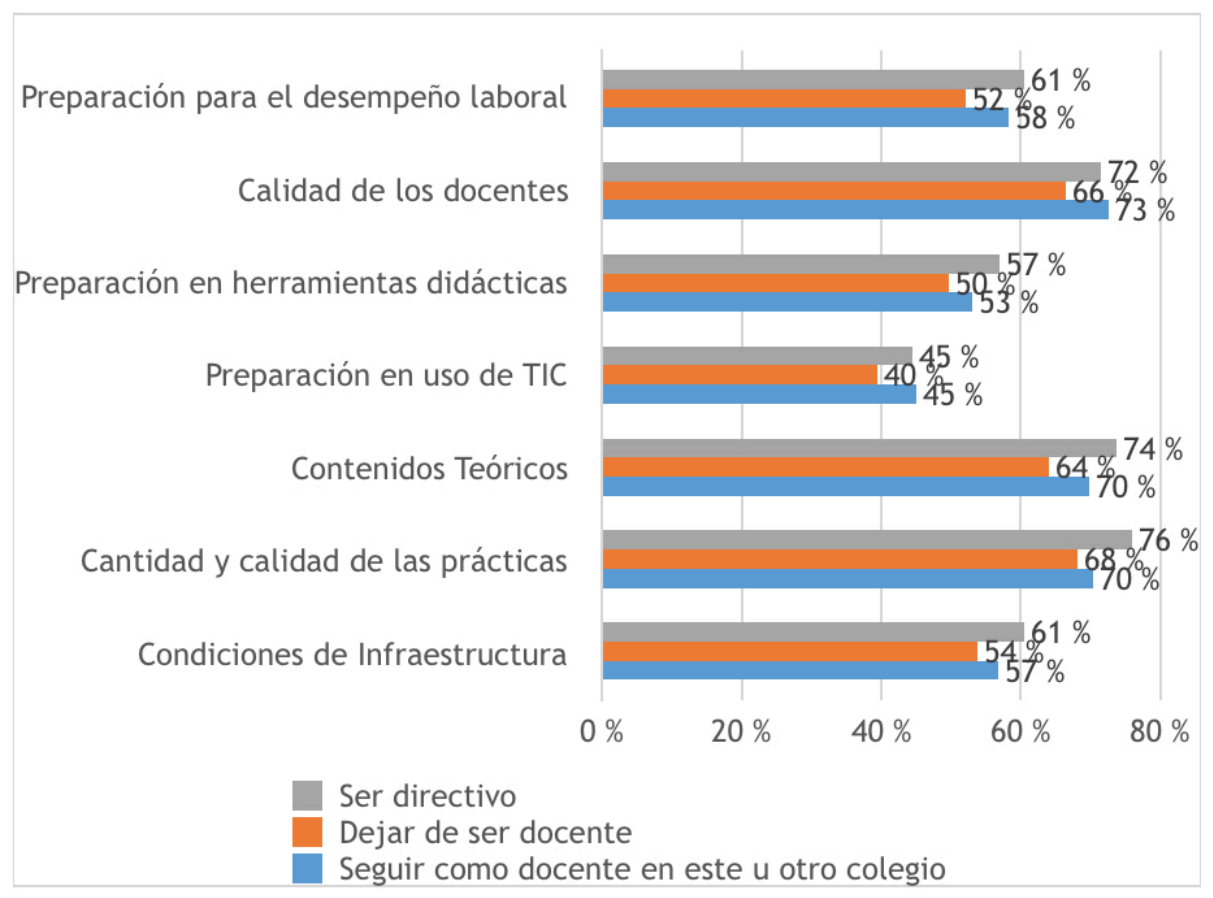

Fig. 13. Evaluación de procesos formativos (sólo respuesta "bueno - muy bueno") segmentados por declaración de expectativas a 10 años más.

“¿Cómo cree que continuará su carrera en 10 años más?” 
su proceso de formación; en cambio, quienes quieren dejar de trabajar como profesores, presentan una valoración menos positiva.

Cerca de un 40\% de los encuestados quiere continuar siendo docente, ya sea en el mismo establecimiento u otro. Mientras, cerca de un 15\% de los docentes encuestados quiere "dejar de trabajar como docente" y un porcentaje similar considera que, en el futuro, ocupará un cargo directivo/a. El resto, estima que en 10 años más estará jubilado/a.

Como se puede apreciar, tanto los docentes que quieren dejar de serlo, como los que quieren continuar en la profesión, evalúan de manera similar sus procesos educativos en todas las dimensiones con una leve tendencia más crítica de docentes que quieren dejar la labor docente.

\section{CONCLUSIONES}

Esta investigación realizada sobre la evaluación que los propios egresados hacen de su formación ofrece elementos útiles para retroalimentar los procesos formativos, al dar cuenta de fortalezas y debilidades percibidas en su Formación Inicial Docente según la selectividad de las instituciones formadoras y la edad de los docentes participantes.

En relación a las instituciones No-Selectivas tienen una visión más critica de sus procesos formativos y sus debilidades se concentran en aspectos referidos a:

- Condiciones de infraestructura.

- Preparación de herramientas didácticas.

- Preparación para el mundo laboral.

En cambio, los egresados de instituciones Selectivas reportan notoriamente, mayores niveles de evaluación positiva cuando se les consulta sobre sus procesos de formación inicial.

Hay varios ítems en donde estas diferencias se matizan (como en la "preparación teórica" o "calidad de los docentes") y en las cuales existe una evaluación transversalmente positiva.

Realizar un análisis diferenciado según la selectividad nos permite inferir que egresados de instituciones Selectivas han sido más beneficiados de las acciones de fortalecimiento de la FID de estas últimas décadas, concentrando sus dificultades en dimensiones que no han sido foco de las iniciativas de mejoramiento (Ruffinelli, 2013).

Uno de los elementos interesantes para analizar, es la percepción mucho más crítica de los profesores jóvenes. Desde nuestra interpretación, tiene que ver con la idea de los docentes que sus procesos formativos podrían haber sido mejores y que le traspasan responsabilidad en las falencias que sintieron al momento de insertarse al mundo laboral a sus universidades de egreso. Contrario a ello, los docentes de más experiencia, por una parte ya asumieron esos aprendizajes, o bien, no consideran que la universidad de egreso tenga responsabilidad en las brechas de su formación pedagógica y lo que el ejercicio laboral les exige. El incorporar a 
docentes con más de 20 años de experiencia (28\% de la muestra), puede explicar en parte, los resultados diferenciados que tuvo esta investigación con Rufinelli (2013).

Por otra parte, en este estudio se buscó rescatar e indagar en las expectativas de los docentes preguntándoles cómo veían su carrera en 10 años más; lo relevante en torno a nuestro objeto de estudio, es que las expectativas de los docentes no tienen vínculo con su evaluación de sus procesos formativos.

En este sentido, suponíamos como hipótesis que los docentes que quieren dejar de trabajar como tales en un futuro cercano, evalúan peor sus procesos formativos que sus pares que se visualizan en el futuro aún como docentes o como directivos de establecimiento. Sin embargo, al contrario de ello, la expectativa de los docentes no genera ninguna tendencia al compararse con la evaluación de los procesos formativos y por tanto, los profesionales que quieren "dejar de trabajar como docentes" evalúan de manera muy similar sus procesos formativos en comparación con el resto de sus colegas.

Lo anterior, lejos de descartar una relación causal entre ambas dimensiones, entrega pistas en torno a que la idea de abandonar la profesión docente que tienen los actuales profesores en ejercicio, no tendría relación alguna con su formación docente universitaria sino, más bien, con condiciones laborales o factores que deben ser analizados con más detalle y profundidad, quedando como un desafío e interrogante para futuros procesos de investigación.

Para la Universidad de Magallanes (UMAG), recoger y analizar información sobre tendencias y fenómenos que se producen en torno a la carrera de los docentes es prioritario. Sobre todo, si consideramos el rol que tiene como casa de estudios en una región extrema y aislada como Magallanes, cuyos egresados son más de la mitad del cuerpo docente en ejercicio (56\%). Por ello, es fundamental para la UMAG que las mallas curriculares de las carreras de pedagogía y el diseño general de los procesos formativos actuales se revisen profundamente desde la perspectiva del desarrollo profesional docente, considerando por sobre todo, la opinión de los profesores en ejercicio, que constituye información fundamental para el análisis de los futuros cambios que debiera tener la FID. Se puede indicar entonces, que los procesos de formación docente deben preparar a los futuros profesores para hacer frente a las problemáticas con las que estos efectivamente se encontrarán en las escuelas y donde algunos nudos críticos han sido especificados en este artículo. Sin embargo, hay que tener presente que por más buena que sea la FID, ésta por sí sola, no puede preparar a los profesores para enfrentar exitosamente cada uno de los desafíos que se le presenten durante su carrera docente (Schleicher, 2014). Y en este sentido la ley 20.903, que crea el sistema de desarrollo profesional docente en el artículo 18 $\mathrm{G}$ define la inducción como "el proceso formativo que tiene por objeto acompañar y apoyar al docente principiante en su primer año de ejercicio profesional para un aprendizaje, práctica y responsabilidad profesional efectivos, facilitando su inserción en el desempeño profesional y en la comunidad educativa a la cual se integra" (Diario Oficial de Chile, abril 2016), la que deberá iniciarse dentro del año escolar en que el profesor ingrese a prestar sus servicios profesionales. 
La indagación de la brecha que implica la manera en cómo los docentes son formados y lo que luego se requiere de ellos en su desempeño concreto, se hace fundamental para que en centros de educación superior tomen nota de estos "shock de realidad", tal como lo menciona Veenman (1984) al conceptualizar ese desfase que emerge de los docentes en ejercicio. Aparece entonces la necesidad de construir programas en la FID que se hagan cargo de los déficit detectados, a partir de la implementación de estrategias efectivas, de análisis y reflexión en los distintos niveles del sistema educativo, incorporándolos activa y formalmente a los procesos formativos de la FID, así como en la educación continua de los profesores en ejercicio. De este modo, se asume que la docencia es "una profesión altamente compleja y desafiante, apoyar su ejercicio y aumentar su valoración para las nuevas generaciones, comprendiendo la misión decisiva que cumple esta profesión en la sociedad" (CPEIP, 2016, p.3)․․

\section{REFERENCIAS}

Avalos, B., \& Aylwin, P. (2005). La inserción laboral de los profesores nuevos en Chile. Paideia, $38,9-27$.

Ávalos, B.. (2010). Formación inicial docente en Chile: Calidad y políticas. En Ecos de la revolución pinguina, Santiago, Chile (pp. 257-284). Santiago de Chile: Centro de Investigación Avanzada en Educación (CIAE).

Ávalos, B. (2014). Initial teachers' education in Chile: tensions between policies of support and control. Estudios pedagógicos (Valdivia), 40(Especial), 11-28.

Bellei, C. (2001). El talón de Aquiles de la reforma: Análisis sociológico de la política de los 90 hacia los docentes en Chile. En S. Martinic \& M. Pardo (Eds.), Economía Política de las Reformas Educativas en América Latina (pp. 227-258). Santiago de Chile: CIDE \& PREAL.

Borko, H., Whitcomb, J., \& Kathryn, B. (2008) Genres of Research in Teacher Education. En M. Cochran-Smith, S. Feiman-Nemser, D. McIntyre \& K. Demers (Eds.), Handbook of Research on Teacher Education Enduring Questions in Changing Contexts (3 ed., pp. 10171045). New York: Routledge.

Boyd, D., Lankford, H., Loeb, S., Rockoff, J., \& Wyckoff, J. (2008). The narrowing gap in New York City teacher qualifications and its implications for student achievement in highpoverty schools. Journal of Policy Analysis Management, 27(4), 793-818.

Brain, K., Reid, I., \& Boyes, L. (2006). Teachers as mediators between educational policy and practice. Educational Studies, 32(4), 411-423.

CINDA (2014). Evaluación del aprendizaje en innovaciones curriculares de la Educación

1 PPT Sistema Nacional de Desarrollo Profesional Docente, Jaime Veas Sánchez, Director CPEIP. En http://www. ucentral.cl/prontus_ucentral2012/site/artic/20160506/asocfile/20160506165206/ppt_sdpd.pdf. Consultado el 3-04-18. 
Superior. Santiago, Chile: Ediciones e Impresiones Copygraph.

Cisternas, T. (2011). La investigación sobre formación docente en chile. Territorios explorados e inexplorados. Calidad en la educación, 35,131-164.

Conway, P. (2013). Cultural flashpoints: The politics of teacher education reform in Ireland. Educational Forum, 77(1), 51-72.

Darling-Hammond, L. (2006). Constructing 21st-century teacher education. Journal of Teacher Education, 57(3), 300-314.

Díaz, C., Solar, M., Soto, V., \& Conejeros del Solar, M. (2015). Formación docente en Chile: percepciones de profesores del sistema escolar y docentes universitarios. Civilizar Ciencias Sociales y Humanas, 15(28), 229-246.

Gaete, A., Gómez, A., \& Bascopé, M. (2016). ¿Qué le piden los profesores a la formación inicial docente en Chile?. Centro de Politicas Publicas UC. 11 (86).

García, R., \& Castro. Z, A. (2012). La formación permanente del profesorado basada en competencias. Estudio exploratorio de la percepción del profesorado de Educación Infantil y Primaria. Educatio Siglo XXI, 30(1), 297-322.

Gentili, P., \& Suárez, D. (2004). Reforma Educativa y Luchas Docentes en América Latina. Educ. Soc., Campinas, 25(89), 1251-1274.

Goldhaber, D. D., \& Brewer, D. J. (2000). Does teacher certification matter? High school teacher certification status and student achievement. Education Evaluation and Policy Analysis, 22, 129-45.

Gorichon, S., Ruffinelli, A., Pardo, A., \& Cisternas, T. (2015). Relaciones entre Formación Inicial e Iniciación profesional de los docentes. Principios y desafíos para la formación práctica. Cuadernos de Educación, 66, 1-20.

Hernández, V., Baptista, P., \& Fernández, C. (2003). Metodología de la Investigación. México: Mc Graw Hill.

Hueso, A., \& Cascanti, M. (2012). Metodología y técnicas cuantitativas de investigación. Cuadernos docentes en procesos de desarrollo Número 1. Valencia: Universitat Politécnica de València.

Kaufman, D., Johnson S., Kardos S., Liu, E., \& Peske H. (2002). “Lost at Sea”. New teachers experiences with curriculum and assessment. Teachers College Record, 104(2), 273-300.

Manzi, J., González, R., \& Sun, Y. (2011). La evaluación docente en Chile. Santiago de Chile: MIDE UC.

Marcelo, C. (2009). Formalidad e informalidad en el proceso de aprender a enseñar Revista de Educación, 350, 31-55.

Ministerio de Educación, Mineduc (n.d.). En Ámbito formación inicial de profesores. Financiamiento institucional. Recuperado de http://www.mecesup.cl/index2.php?id_ contenido $=15048 \& i d \_$portal $=59 \& i d \_$seccion $=3606$

Musset, P. (2010). Initial teacher education and continuing training policies in a comparative perspective: Current practices in OECD countries and a literature review 
on potential effects. OECD Education Working Papers, N.48. OECD Publishing. doi: $10.1787 / 19939019$

National Council for Accreditation of Teacher Education (NCATE) (2010). Transforming teacher education through clinical practice: A national strategy to prepare effective teachers. Report of the Blue Ribbon Panel on Clinical Preparation and Partnerships for Improved Student Learning. Washington, DC: NCATE. Última visita 5 de marzo de 2011. Recuperado desde: http://www.ncate. org/LinkClick.aspx? leticket=zzeiB1OoqPk\%3D ytabid=715

Nicholl, B., \& R. McLellan (2008). Were all in the game whether we like it or not to get a number of As to Cs. Design and technology teachers struggles to implement creativity and performativity policies. British Educational Research Journal, 5 (5), 585-600.

OCDE (2012), Equity and Quality in Education: Supporting Disadvantaged Students and Schools (Equidad y calidad en la educación: Apoyando a estudiantes y colegios de estratos más bajos), OCDE Publishing, Paris, http://dx.doi. org/10.1787/9789264130852-en.

Ortúzar, S., Flores, C., Milesi, C., \& Cox, C. (2009). Aspectos de la formación inicial docente y su influencia en el rendimiento académico de los alumnos. Santiago de Chile: Camino al Bicentenario: Propuestas para Chile.

Park, H., \& Byun, S.-Y. (2015). Why Some Countries Attract More High-Ability Young Students to Teaching: Cross-National Comparisons of Students' Expectation of Becoming a Teacher. Comparative Education Rewiew, 59(3), 523-549.

Poppleton, P., \& J. Williamson (2004). New Realities of Secondary Teachers' Work Lives. Oxford: Symposium Books

Puga, I., Polanco, D., \& Corvalán, D. (2015). Segregación de la formación y carrera docente y su rol en la reproducción social de la desigualdad. Calidad en la educación, (43), 57-102.

Rivkin, S., Hanushek, E., \& Kain, J. (2005); Teachers, schools, and academic achievement. Econometrica, 73(2), 417-458.

Rockoff, J. (2004) “The Impact of Individual Teachers on Student Achievement: Evidence from Panel Data." American Economic Review, 94(2), 247-252.

Ruffinelli, A. (2013). La calidad de la formación inicial docente en Chile: la perspectiva de los profesores principiantes. Calidad en la educación, 39, 117-154.

Sanders, W. L., \& Rivers, J. C. (1996). Cumulative and residual effects of teachers on future student academic achievement. Knoxville: University of Tennessee Value-Added Research and Assessment Center.

Schleicher, A. (2014). Equity, Excellence and Inclusiveness in Education: Policy Lessons From Around the World (Equidad, excelencia e inclusividad en la educación: Lecciones de políticas elaboradas en distintas partes del mundo). Paris: OCDE Publishing.

Tenti, E. (2005). La Condición Docente. Análisis Comparado de la Argentina, Brasil, Perú y 
Uruguay. Buenos Aires: Siglo Veintiuno Editores.

Tenti, E. (2007). Consideraciones sociológicas sobre profesionalización docente. Educación y Sociedad, 28(99), 335-353.

Veenman, S. (1984) Perceived Problems of Beginning Teachers. Review of Educational Research, 54(2), 143-178. 